\title{
Vinagre artesanal como impacto socioeconómico medio educativo.
}

The socio-economic impact of artisanal vinegar as an educational tool.

Velasteguí López Efraín. ${ }^{1}$ \& Rodríguez Lirio Fabián. ${ }^{2}$

\section{DOI: https://doi.org/10.33262/visionariodigital.v1i1.238}

\section{Resumen.}

El vinagre es una solución diluida de ácido acético producido por fermentación, a la que se le agregan sales y extractos de otras materias, en la primera fermentación es la alcohólica, que es llevada a cabo por la acción de fermentos que transforman el azúcar en alcohol. Y la segunda es la acética en la que intervienen un grupo amplio de acto bacterias que tienen el poder de combinar el oxígeno con el alcohol para formar ácido acético.

A partir de ahí se inicia el proceso de crianza, para lograr el pleno desarrollo de los aromas y obtener un máximo grado de calidad.

El vinagre destinado al consumo se pasa a las plantas embotelladoras, donde su grado de acidez debe ser, como mínimo de 6 grado de acidez, el vinagre es un conservante natural de los alimentos y se utiliza en la industria alimenticia por tener la propiedad de reducir el pH de los alimentos para evitar el crecimiento de bacterias.

Es un excelente ingrediente para marinar ya que es un ablandador natural que desdobla las fibras y proteínas de las carnes, también ayuda a resaltar el sabor y se utiliza en la cocina para salsas, mayonesas e incluso guisos porque aporta una acidez muy interesante que se utiliza como agente antibacteriano porque ayuda a eliminar bacterias que pueden ser dañinas a la salud. Evita el crecimiento de hongos, desinfecta los equipos que se utilizan para procesar alimentos y neutraliza los malos olores característicos de ciertos alimentos.

Palabras clave: impacto, vinagre artesanal, mercado, socio económico.

\section{Abstract.}

${ }^{1}$ Universidad Técnica de Ambato, Ambato, Ecuador, le.velastegui@uta.edu.ec ${ }^{2}$ Universidad Técnica de Cotopaxi.Cotopaxi.Ecuador, fabian19871930@gmail.com 
Vinegar is a diluted solution of acetic acid produced by fermentation, to which are added salts and extracts of other materials, in the first fermentation is the alcoholic, which is carried out by the action of ferments that transform sugar into alcohol. And the second is the acetic in which involved a broad group of act bacteria that have the power to combine oxygen with alcohol to form acetic acid.

From there the process of aging begins, to achieve the full development of the aromas and obtain a maximum degree of quality.

The vinegar intended for consumption is passed to the bottling plants, where their degree of acidity must be at least 6 degrees of acid. Vinegar is a natural preservative of foods and is used in the food industry because it has the property of reduce the $\mathrm{pH}$ of food to prevent the growth of bacteria.

It is an excellent ingredient for marinating since it is a natural softener that unfolds the fibers and proteins of the meats. It also helps to highlight the flavor and be used in the kitchen for sauces, mayonnaise and even stews because it provides a very interesting acidity that is used as an antibacterial agent because it helps to eliminate bacteria that can be harmful to health. It prevents the growth of fungi, disinfects the equipment used to process food and neutralizes the bad odors characteristic of certain foods.

Keywords: impact, artisanal vinegar, market, economic partner.

\section{Introducción.}

\section{Vinagre natural.}

Segun los autores Ramsar M. 2014., Hearn S. 2016., Gertler P. 2014., Prak S. 2015., Morales D. 2015., Ghosh S. 2014. \& Budak N. 2014. afirman que el vinagre es un excelente conservante, y un ingrediente imprescindible en la preparación de sabores y aliños, siendo También necesario para la preparación de carnes, el vinagre es un condimento líquido, que se obtiene por la fermentación de alcohólica, que, durante ese proceso, se transforman en ácido acético.

Además de su uso gastronómico, el vinagre puede usarse en el ámbito de la medicina y la enfermería para curaciones de la piel gracias a su alto nivel de acidez y hasta para la limpieza hogareña sobre todo de vidrios y cristales.

El vinagre es una solución diluida de ácido acético que se puede elaborar a partir de casi cualquier fuente rica en carbohidratos por medio de un proceso de fermentación en dos pasos: fermentación alcohólica y acética. Aparte de ácido acético, el vinagre contiene otras sustancias en menor proporción que influyen en sus características organolépticas y 
en el contenido de ácido. No se considera un alimento, pues no tiene gran valor nutricional, sin embargo, es muy usado como agente saborizante y conservante.

Fig 1. Vinagre de piña

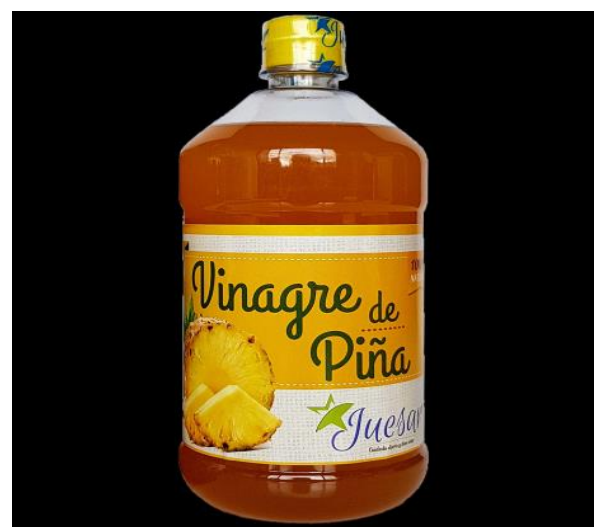

Fuente:https://www.google.com.ec/search?q=vinagre\&rlz=1C1SQJL_esEC807 EC807\&source $=$ lnms\&tbm $=i s c h \& s a=X \& v e d=0 a h U K E w j v 5$ urC9ZjeAhVqTd8K HZ96DuAQ_AUIDigB\&biw=1536\&bih=754\#imgrc=hskCM0DWLNTp-M:

El vinagre es una solución diluida de ácido acético obtenida por un proceso fermentativo de dos etapas. En la primera etapa una solución inocua de azucares y/o sustancias fermentables, son convertidos en etanol mediante levaduras, mientras que, en la segunda fase, la solución alcohólica producida es oxidada hasta ácido acético por la acción de bacterias, este proceso se denomina acetificación.

Fig 2. Vinagre de manzana

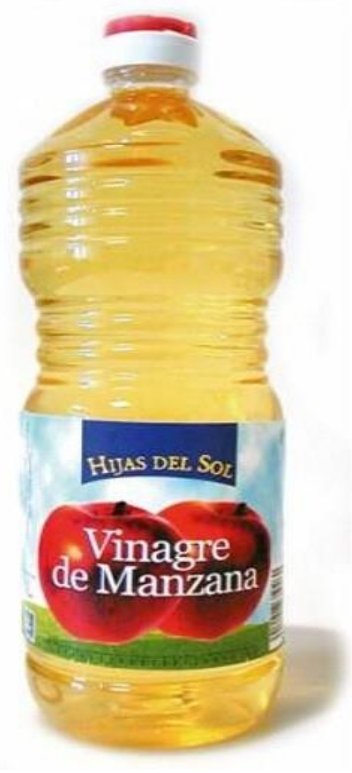

Fuente:ttps://www.google.com.ec/search?q=vinagre\&rlz=1C1SQJL_esEC8 07EC807\&source $=$ lnms\&tbm $=$ isch \&sa=X\&ved=0ahUKEwjv5urC9ZjeAhV qTd8KHZ96DuAQ_AUIDigB\&biw=1536\&bih=754\#imgrc=XN1GkvHIEN L6VM: 
El mercado de elaboración y venta de vinagre en el ecuador aún no se encuentra saturado pero cada vez son más las empresas que ven una oportunidad, es por eso que se realizó un análisis de mercado con el estudio de los competidores directos e indirectos para el vinagre natural de guineo.

Fig 3. Vinagre de guineo.

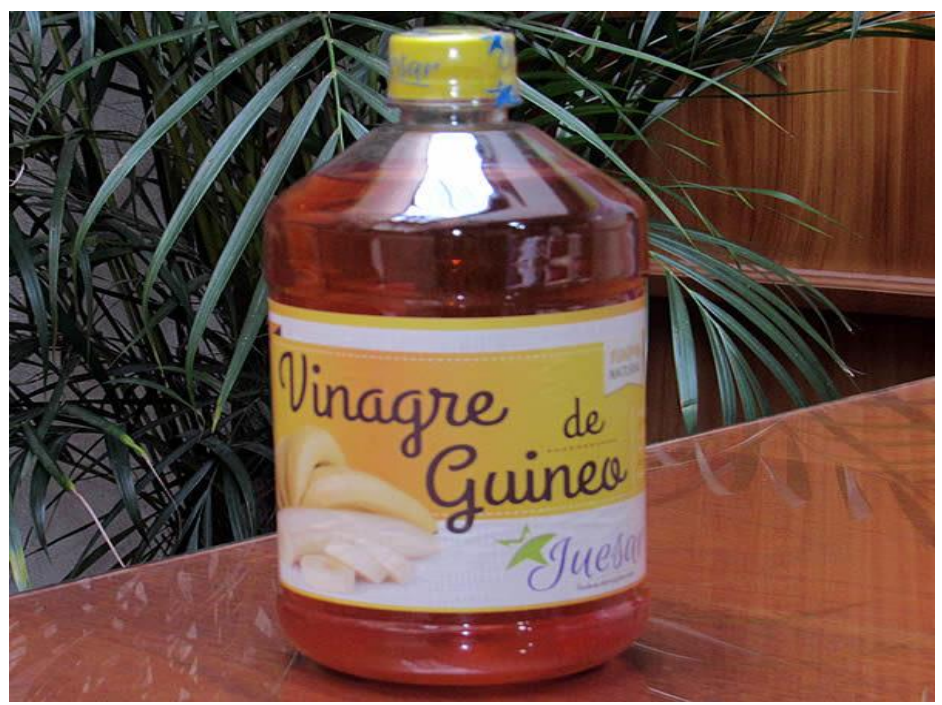

Fuente:https://www.google.com.ec/search?q=vinagre+de+guineo\&rlz=1C1SQJ $\underline{\mathrm{L}}$ esEC807EC807\&source $=\operatorname{lnms\& tbm=isch\& sa=X\& ved=0ahUKEwjXu-}$ f8iJneAhVjTt8KHXt1C7wQ_AUIDigB\&biw=1536\&bih=754\&dpr=1.25\#imgrc $=\mathrm{qxbocwRmQlwEXM:}$

Los países europeos cumplen un rol importante en cuanto al consumo del vinagre de frutas producidos por los países latinoamericanos, pues si bien ellos son los principales son los principales productores de vinagre, en el caso de hablar de frutas estos países no tienen materia prima necesaria para realizar este producto. En España y Alemania se produce gran cantidad de vinagre tradicional, la principal producción europea de vinagre es Alemania.

\section{Impacto.}

Según los autores Ramírez I. 2016., Ferreyra M. 2014., Romo S. 2014., Vallejo L. 2016., Davies C. 2015. \& Prada D. 2015. Afirman que las empresas desarrollan estrategias de mercado para ayudar a desarrollar identidades de marca, aumentar las ventas y ganar cuota de mercado. Los enfoques estratégicos incluyen un énfasis en herramientas de promoción a disposición de especialistas en mercado y el uso de estas herramientas tiene un impacto en el rendimiento de la empresa. El objetivo de una estrategia de mercado esta en los efectos de los elementos específicos y como afectan a la posición de una empresa en el mercado. La compresión de como las herramientas y elementos de trabajo estratégicos ayudan a las empresas a implementar estrategias de mercado que aumentan los beneficios.

El impacto que podemos tener en hacer conocer nuestro producto puede ser bueno como malo, debemos hacerlo conocer de la mejor manera, tener una buena presentación del mismo para así tener buena acogida del producto. 
La siguiente investigación es para dar a conocer que la mercadotecnia es mucho más que vender un producto al consumidor, sino que también implica ser socialmente responsable a través de análisis y estudios para asi cuidar al medio ambiente y tener una mejor visión del impacto en las personas en su vida cotidiana para hacer cambiar su manera de pensar, desarrollar nuevos y mejores criterios de compra y que a la vez las empresas generen dinero y sean rentables. Nuestro objetivo es mostrar también que es posible obtener beneficios siendo una empresa responsable con su entorno a la hora de producir y distribuir sus productos pues como a continuación lo expondremos son cada vez mas las personas que eligen un producto verde por parte de empresas que se preocupan por el bienestar de la sociedad y su preservación.

Los estudios de impacto social guardan una estrecha relación con los estudios de impacto ambiental, impactos biofísicos. Por ejemplo: ambos impactos suelen clasificarse en un rango que va de lo deseable hasta lo adverso positivo y negativo, tener una escala, una duración en tiempo y espacio corto, mediano o largo plazo, local, regional o internacional. Durante años la medición de impacto social ha sido utilizada en los campos del desarrollo y la cooperación internacional, la política y las obras filantrópicas únicamente. Los términos evaluación y seguimiento se utilizaban para medir el impacto de un programa/proyecto en la población. La evaluación /seguimiento servía como justificante de subvenciones y donaciones, así como para garantizar que los objetivos definidos por las mismas eran alcanzados. Con la aparición de un nuevo modelo basado en la economía social y el cuarto sector empresas de triple resultado se produjo un cambio de paradigma. La prioridad para las empresas sociales o es únicamente el beneficio económico. Sino que también quieren alcanzar el objetivo de intentar generar impacto social. Este nuevo objetivo planteo la cuestión de su medición. ¿cómo pueden medir las empresas sociales su propio impacto en la sociedad?

En la evaluación de impacto, se reconocen cuatro dimensiones que definen a una amplia variedad de tipos de estimaciones de impacto. Una primera dimensión se refiere al tipo de variable sobre la que se mide el impacto del programa. Según esto, se puede clasificar a las investigaciones en cuantitativas o cualitativas. Son cuantitativas si la variable a medir es susceptible de ser descrita en forma métrica; por ejemplo: rentabilidad de la explotación. Las investigaciones cualitativas se enfocan en variables a las que no se puede aplicar una métrica.

\section{Mercado.}

Según los autores European C. 2015., Pro C. 2011., Romo S. 2014. \& Vallejo L. 2016. afirman que el mercado también es el ambiente social o virtual que propicia las condiciones para el intercambio. En otras palabras, debe interpretarse como la institución u organización social a través de la cual los ofertantes productores o vendedores y demandantes consumidores o compradores de un determinado tipo de bien o de servicio, entran en estrecha relación comercial a fin de realizar abundantes transacciones comerciales. 
Fig 4. Mercado del vinagre

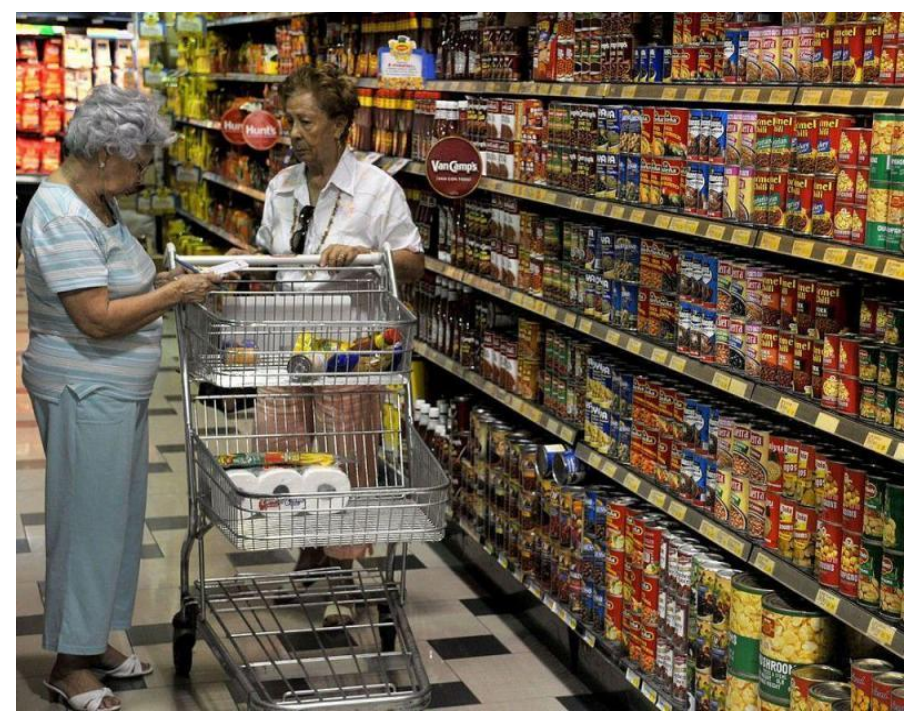

Fuente:https://www.google.com.ec/search?q=mercado+del+vinagre \&rlz=1C1S QJL_esEC807EC807\&source $=$ lnms\&tbm $=$ isch\&sa $=$ X\&ved=0ahUKEwjgzeH05 JveAhWETd8KHZcmAVUQ_AUIDigB\&biw=1536\&bih=754\#imgrc=Cj5I0qX G3YM7qM:

El mercado es un mecanismo a través del cual compradores y vendedores interactúan para determinar precios e intercambiar bienes y servicios.

En el caso de la cadena de producción involucrada en el mercado de vinagre natural de guineo, esta comprende a: la agricultura orgánica, empresas productoras de alimentos naturales procesados, restaurantes vegetarianos, naturistas, macrobióticos, distribuidores de alimentos naturales procesados, tiendas naturistas y de abarrotes y supermercados. El mercado de elaboración y venta de vinagres en el Ecuador aun no se encuentra saturado pero cada vez son mas las empresas que ven una oportunidad, es por esto que se realizó un análisis de mercado con el estudio de los competidores directos e indirectos para el vinagre natural.

Fig 5. Mercado del vinagre

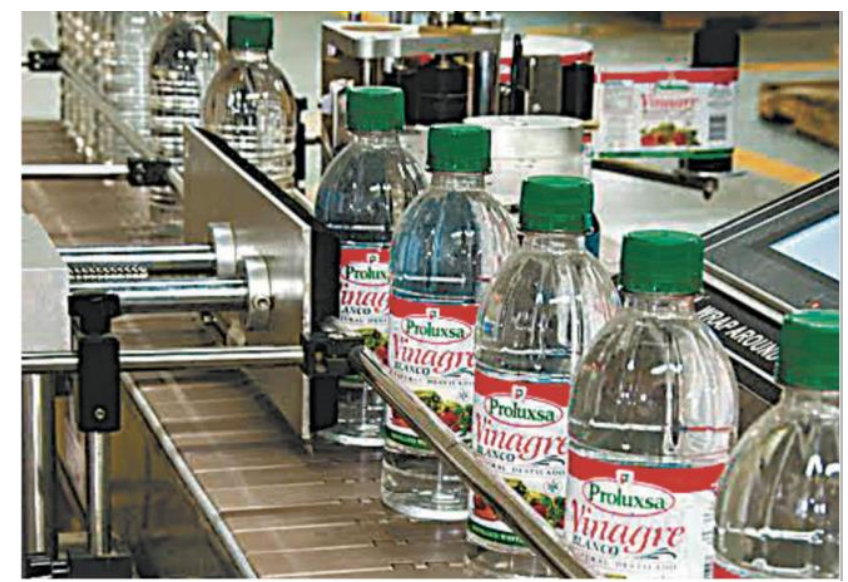

Fuente:https://www.google.com.ec/search?rlz=1C1SQJL_esEC807EC807\&biw= 1536\&bih=754\&tbm=isch\&sa=1\&ei=EK OW6rFFfG2ggfI Yf4Dg\&q=vinagre+ 
conquista+mercado\&oq=vinagre+conquista+mercado\&gs_l=img.3...113005.1229 88.0.123485.52.32.0.2.2.0.315.4739.0j23j2j1.26.0....0...1c.1.64.img..27.11.1829.0 ..0j0i67k1.0.It_4RVi_wUM\#imgdii=izwjPAkAwKJRM:\&imgrc=utdZsao0JltuCM:

En Ecuador existe una diversidad de vinagres, sin embargo a pesar de ser un país que cuenta con un sin número de frutas exóticas, dentro de la producción de vinagres no sobresale alguno que sea elaborado a partir de estas, en la provincia de Manabí se produce vinagre artesanal de banano, que es usado para darle un sabor diferente a comidas, encurtidos e incluso en el ají como un buen preservante, sin embargo no cumple con las características de calidad necesarias, además de no seguir un proceso de doble fermentación.

\section{Socioeconómico.}

Según los autores Castro R. 2014., Martínez R. 2013., Vera O. 2013., Gil J. 2014. \&

Niklas S. 2015. Afirman que el nivel o estatus socioeconómico es una medida total económica y sociológica combinada de la preparación laboral de una persona, de la posición económica y social individual o familiar en relación a otras personas, basada en sus ingresos, educación, y empleo. Al analizar el nivel socioeconómico de una familia se analizan, los ingresos dell hogar, los niveles de orientación educación, y ocupación, como también el ingreso combinado, comparado con el individual, son analizados los atributos personales de sus miembros.

Fig 6. Nivel socioeconómico

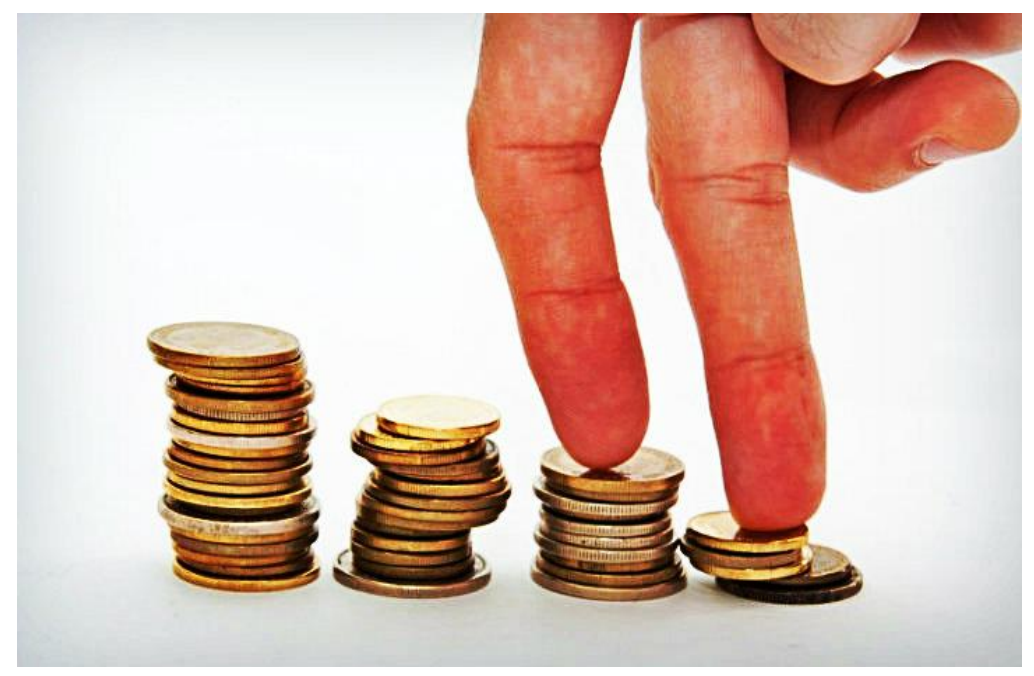

\section{Fuente:}

https://www.google.com.ec/search?rlz=1C1SQJL_esEC807EC807\&biw=1536\& bih=754\&tbm=isch\&sa=1\&ei=EK_OW6rFFfG2ggfI_Yf4Dg\&q=socioeconomic o\&oq=socioec\&gs_l=img.1.0.0110.2431.8282.0.9882.34.16.0.0.0.0.274.1613.3j7 j1.11.0...0...1c.1.64.img..25.8.1359.0..0i24k1j0i67k1.0.MD2igxouOLk\#imgrc= Q7lcz-1XuP5L3M: 
Se clasifica por lo general en tres categorías, alto, medio y bajo en las cuales una familia puede ser ubicada. Para ubicar a una familia o individuo en una de estas tres categorías se toman en cuentas las siguientes variables ingreso, educación y ocupación, pueden ser analizadas o procesadas por alguien.

El concepto de economía social, y de las empresas que la conforman, ha ido evolucionando reflejando los cambios económicos y sociales. Sin embargo, a pesar del esfuerzo tanto de la comunidad investigadora como de las autoridades legislativas en definir la economía social, existe aún un importante desconocimiento acerca de su impacto sobre la economía y sobre la sociedad en un conjunto. Esta falta de valoración se explica, en parte, por la dificultad de hacer visible en términos numéricos su aportación a la sociedad en comparación con la aportación de otros agentes económicos, en especial de las empresas tradicionalmente enmarcadas en el sector privado. Los valores propios que comparten las empresas de economía social motivan un comportamiento diferenciado de esas empresas en relación con la composición de sus plantillas, las condiciones de trabajo, su especialización productiva y su ubicación geográfica.

Fig 7. Nivel socioeconómico

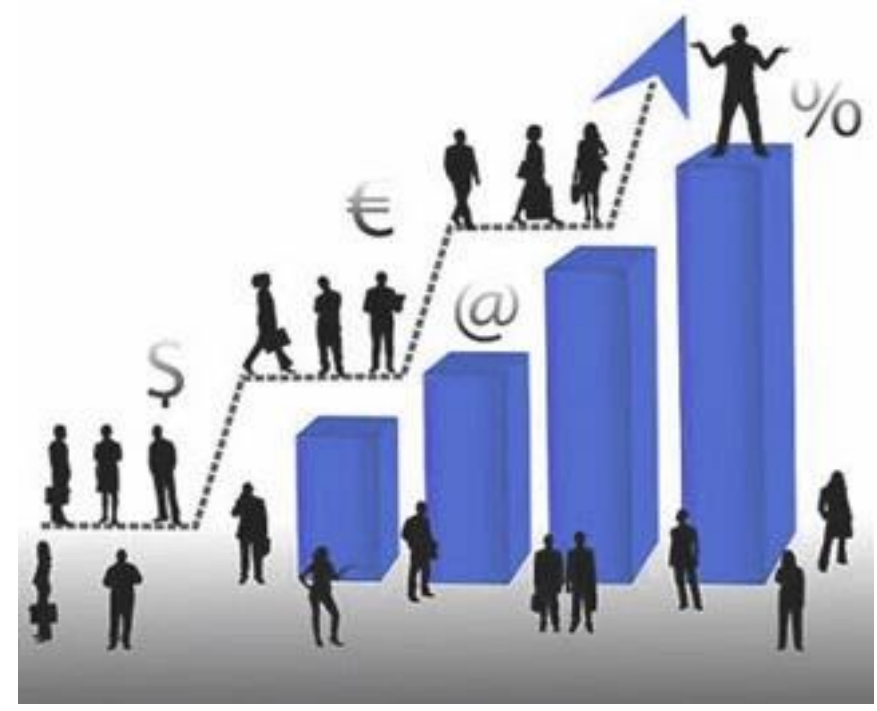

Fuente:https://www.google.com.ec/search?rlz=1C1SQJL_esEC807EC807\&biw $=1536 \&$ bih=754\&tbm=isch\&sa=1\&ei=EK_OW6rFFfG2ggfI_Yf4Dg\&q=socioe conomico\&oq=socioec\&gs_l=img.1.0.0110.2431.8282.0.9882.34.16.0.0.0.0.274. 1613.3j7j1.11.0...0...1c.1.64.img..25.8.1359.0..0i24k1j0i67k1.0.MD2igxouOLk \#imgrc=nEV011iLrsgf0M:

\section{Conclusiones.}

- Es un ingrediente perfecto para aderezar la tradicional cocina mediterránea, especialmente para ensaladas y además es un elemento fundamental del preservar alimentos.

- Se puede añadir a las ensaladas proporcionándoles un toque a manzana que se combina muy bien con pescados, salsas suaves y carnes blancas. 
- Es conveniente que el envasado se realice en frascos de vidrio a temperaturas no menor de $85^{\circ} \mathrm{C}$ y sellados herméticamente.

\section{Referencias.}

Budak, N. (2014). Recuperado el 15 de 10 de 2018, de http://www.mizkan.com/Downloads/Functinal-Properties-of-Vinegar.aspx

Davies, C. (2015). Recuperado el 15 de 10 de 2018, de https://riunet.upv.es/bitstream/handle/10251/59399/DAVIES\%20-

$\% 20$ Estudio\%20de\%201os\%20procesos\%20biotecnol\%C3\%B3gicos\%20de\%20acetific aci\%C3\%B3n\%20para\%20la\%20producci\%C3\%B3n\%20de\%20vinagre\%20d....pdf?se quence $=1$

European, C. (2015). Recuperado el 15 de 10 de 2018, de file://C:/Users/ZBook/Downloads/Vinegar\%20RR\%20Jul\%202015.pdf

Ferreyra, M. (2014). Recuperado el 15 de 10 de 2018, de http://www.redalyc.org/pdf/145/14532635007.pdf

Gertler, P. (2014). Recuperado el 15 de 10 de 2018, de http://www.iica.int/sites/default/files/events/presentations/201609/evaluacion_de_impacto_inia_1_.pdf

Ghosh, S. (2014). Recuperado el 15 de 10 de 2018, de http://www.scielo.br/pdf/bjce/v29n3/03.pdf

Hearn, S. (2016). Recuperado el 15 de 10 de 2018, de https://www.odi.org/sites/odi.org.uk/files/odi-assets/publications-opinionfiles/10302.pdf

Morales, D. (2015). Recuperado el 15 de 10 de 2018, de http://www.redalyc.org/pdf/654/65452535006.pdf

Prada, D. (2015). Recuperado el 15 de 10 de 2018, de http://bdigital.unal.edu.co/50786/1/1032440911.2015.pdf

Prak, S. (2015). Recuperado el 15 de 10 de 2018, de https://madrid.impacthub.net/wp-content/uploads/2017/10/gu\%C3\%ADamedici\%C3\%B3n-de-impacto-esp-1.pdf

Pro, C. (2011). Recuperado el 15 de 10 de 2018, de https://www.prochile.gob.cl/wpcontent/files_mf/documento_09_06_11155755.pdf

Ramirez, I. (2016). Recuperado el 15 de 10 de 2018, de http://www.web.facpya.uanl.mx/vinculategica/Revistas/R2/1024-1039\%20$\% 20$ Impacto $\% 20 \mathrm{de} \% 201 \mathrm{a} \% 20$ mercadotecnia\%20social\%20en $\% 20$ empresas $\% 20 \mathrm{y} \% 20 \mathrm{e}$ $1 \% 20$ consumidor\%20en\%20Moxico.pdf

Ramsar, H. (2014). Recuperado el 15 de 10 de 2018, de https://www.ramsar.org/sites/default/files/documents/pdf/lib/hbk4-16.pdf

Romo, S. (2014). Recuperado el 15 de 10 de 2018, de http://dspace.udla.edu.ec/bitstream/33000/2153/1/UDLA-EC-TIAG-2011-19.pdf 
Romo, S. (2014). Recuperado el 15 de 10 de 2018, de http://dspace.udla.edu.ec/bitstream/33000/2153/1/UDLA-EC-TIAG-2011-19.pdf

Vallejo, L. (2016). Recuperado el 15 de 10 de 2018, de http://repositorio.puce.edu.ec/bitstream/handle/22000/11592/Tr\%C3\%A1bajo\%20de\%2 0titulaci\%C3\%B3n\%20-\%20Liliana\%20Vallejo.pdf?sequence $=1$

Vallejo, L. (2016). Recuperado el 15 de 10 de 2018, de http://repositorio.puce.edu.ec/bitstream/handle/22000/11592/Tr\%C3\%A1bajo\%20de\%2 0titulaci\%C3\%B3n\%20-\%20Liliana\%20Vallejo.pdf?sequence $=1$

Castro, R. (2014). Recuperado el 22 de 10 de 2018, de http://www.redalyc.org/pdf/174/17429940002.pdf

Gil, J. (2014). Recuperado el 22 de 10 de 2018, de http://www.revistaeducacion.mec.es/doi/362_162.pdf

Martinez, R. (2013). Recuperado el 22 de 10 de 2018, de https://repositorio.cepal.org/bitstream/handle/11362/4070/1/S2013075_es.pdf

Niklas, S. (2015). Recuperado el 22 de 10 de 2018, de https://www.researchgate.net/publication/273307259_What_is_Socioeconomics_An_O verview_of_Theories_Methods_and_Themes_in_the_Field

Vera, O. (2013). Recuperado el 22 de 10 de 2018, de file://C:/Users/ZBook/Downloads/DialnetEvaluacionDelNivelSocioeconomicoPresentacionDeUnaE-4262712.pdf

\section{Para citar el artículo indexado.}

Velasteguí E, \& Rodríguez F. (2017). Vinagre artesanal como impacto socioeconómico medio educativo. Revista electrónica Visionario Digital 1(1), 74-83. Recuperado desde: http://www.visionariodigital.org/index.php/VISIONARIODIGITAL/article/view/40/47

\section{Ciencia LDigital}

El artículo que se publica es de exclusiva responsabilidad de los autores y no necesariamente reflejan el pensamiento de la Revista Ciencia Digital.

El articulo queda en propiedad de la revista y, por tanto, su publicación parcial y/o total en otro medio tiene que ser autorizado por el director de la Revista Ciencia Digital.
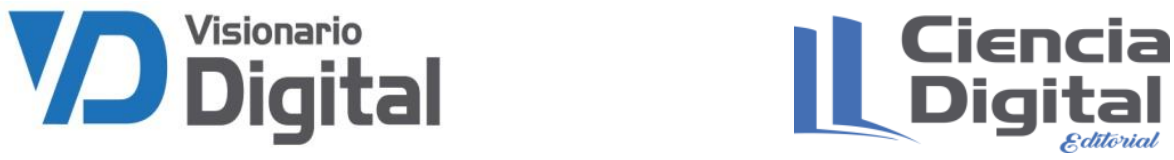